\title{
Prevalência de tabagismo na Universidade Federal de São Paulo, 1996 - dados preliminares de um programa institucional
}

\author{
S.A. Ribeiro, J.R. de B. Jardim, R.R. Laranjeira, A.K.S. Alves, F. Kesselring, L. Fleissig, M.Z.h. \\ ALmeida, M. MATSUDA, R.S. HAMAMOTO \\ Trabal horealizado pel o Departamento de Medicina Preventiva, Clínica Médica ePsiquiatria da UniversidadeF ederal deSãoPaulo, São \\ Paulo, SP.
}

RESUMO - OвJ ETIVo. O conhecimento da prevalência do tabagismo é necessário para a realização de programas institucionais adequados que visem a diminuição do número de fumantes. 0 objetivo do trabalho foi verificar a prevalência do tabagismo entre funcionários, docentes, enfermeiros e alunos da Universidade Federal de São Paulo e a aceitabilidade de um programa antifumo.

Pacientes e Métodos. Foram analisadas as respostas obtidas a partir de um questionário contendo 51 questões, distribuídos para as diferentes categorias.

Resultados. A porcentagem total de questionários respondidos foi de $48,6 \%$ (2.613) sendo $37,3 \%$ para funcionários, $49,0 \%$ para docentes, $52,7 \%$ para enfermeiras e $76,5 \%$ para alunos. Verificou-se que a porcentagem total de fumantes na UNIF E SP foi de $15,5 \%$, sendo $23,7 \%$ entre funcionários, $18 \%$ entre docentes, $16 \%$ entre enfermeiros e $8,6 \%$ entre alunos. A faixa etária de maior prevalência

\section{INTRODUÇÃO}

A partir da Primeira Grande Guerra Mundial, iniciou-se um aumento considerável de consumo de tabaco na forma de cigarro, sendo que o consumo por pessoa cresceu rapidamente durante a primeira metade deste século, à taxa de 5 a $15 \%$ ao ano. Na década de 1950 foi publicado o primeiro relato científico relacionando tabagismo com câncer de pulmão. Nos Estados Unidos, o consumo de cigarros atingiu o pico em 1963. Em 1964, a publicação do primeiro relato do "Surgeon General" sobre Tabagismo e Saúde, concluiu que o tabagismo está causalmente relacionado ao câncer de pulmão e laringe nos homens, causa a bronquite crônica e está associado ao risco aumentado de várias outras doenças, incluindo a doença das artérias coronarianas ${ }^{1,2}$. Após esse relato, foi lançada a "Campanha anti-tabagismo", com esforços da saúde pública e das políticas de saúde para educação dos fumantes sobre os riscos pertinentes de fumantes foi a de 31 a 40 anos $(26,6 \%)$. Não houve diferença estatística entre a prevalência de fumantes entre homens e mulheres. $\mathrm{E} m$ ambos os sexos, para todas as faixas etárias, os indivíduos com maior nível de instrução fumavam menos. Das pessoas que responderam o questionário, $82,5 \%$ estavam preocupadas em serem fumantes passivas. Dos fumantes, $55 \%$ estão pensando em largar de fumar e $42 \%$ julgam precisar de algum tipo de ajuda.

Conclusão. Programas educacionais e de cessação do tabagismo em nível institucional são necessários e bem aceitos na UNIFE SP, devendo ser realizados para que as leis de restrição do fumo dentro da instituição sejam efetivamente cumpridas.

UnITERmos: Tabagismo. Prevalência. Funcionários da saúde, docentes, alunos e enfermeiros.

ao consumo de cigarros, assim como expansão do conhecimento científico acerca das conseqüências do tabagi smo para a saúde, incl usive relacionadas aos indivíduos expostos ao fumo passivo.

As campanhas anti-tabágicas nos Estados Unidos têm al terado os contornos da epidemia, verificando-se uma redução da prevalência de fumantes de $41 \%$ em 1965 para 24\% em 1996, 9\% entre médicos e menos de $1 \%$ entre médicos-pneumologistas ${ }^{3-6}$. No Brasil a prevalência de fumantes é de $32,6 \%$, chegando a $40 \%$ na Região $\mathrm{Sul}^{7}$, com cerca de $20 \%$ de médicos fumantes e $8 \%$ de pneumologistas fumantes ${ }^{8}$.

Este fato levou o cartel do tabaco a expandir suas atividades para o terceiro mundo, onde o consumo cresce. No Brasil, o consumo de cigarros cresceu até $7 \%$ ao longo da década de 1970, mas reduziu este ritmo a partir de 1980, fazendo com que o consumo "per capita" venha caindo ao redor de $1 \%$ ao ano na população com 15 ou mais anos de idade ${ }^{9}$. 
E mbora sejam necessárias políticas mais abrangentes e rigorosas, as campanhas anti-tabagismo, no geral, têm influenciado o comportamento dos fumantes. Por outro lado, o tabagismo continua sendo importante problema de saúde pública, sendo a principal causa de morte prevenível nos Estados Unidos. Na rede SUS do E stado de São Paulo, $31 \%$ dos gastos com internações hospitalares se devem à doenças tabaco-associadas; mais de $90 \%$ destas representadas pelas cardiovasculares erespiratórias ${ }^{10}$. Estudos no Rio Grande do Sul, na década de 1980, mostraram prevalência tabágica de $29 \%$ para médicos, $40 \%$ para outros profissionais liberais, 56\% para a população adulta masculina em geral, $67 \%$ para mineradores de carvão e $69 \%$ para plantadores de cana-de-açúcar. Entre alunos de 1ㅇ. grau em Porto Alegre, verificou-se uma percentagem de fumantes quase duas vezes maior em escolas municipais, pobres, do que em escol as particulares, ricas. Em 1991, no Rio Grande do Sul, a prevalência de fumantes em maiores de 18 anos de idade foi de $39,8 \%^{11}$.

No entanto, verificamos no Brasil uma grande carência de estudos que possibilitem conhecer a prevalência real do tabagismo, relacionado aos indicadores sócio-epidemiológicos e comportamentais, sobretudo no ambiente de trabal ho e que consigam, inclusive, usar estes dados para multiplicar campanhas institucionais.

Assim sendo, é papel das universidades criar mecanismos educativos e servir de exemplo para as suas comunidades, tomando a frente numa campanha de redução do tabagismo, apoiando a legislação. A Organização Mundial da Saúde, considera que o alvo prioritário da ação antifumo em países subdesenvolvidos deve ser centrado nos profissionais de saúde ${ }^{12}$.

$\mathrm{Na}$ Universidade F ederal de São Paulo, em São Paulo, Capital, promoveu-se inicialmente um levantamento entre funcionários e alunos para se conhecer qual a prevalência do tabagismo na área de saúde entre diferentes categorias, verificando qual a predisposição dos fumantes em parar de fumar e como as pessoas se preocupam com o tabagismo passivo.

\section{CASUÍSTICA E MÉTOdo}

O complexo UNIFESP (U niversidade Federal de São Paulo - Escola Paulista de Medicina), consta de um conjunto de vários prédios, o do Hospital São Paulo, anexos e cerca de 100 casas local izados na Vila Clementino, São Paulo, Capital. Dividiuse a população de cerca de 5.810 pessoas da U niversidade Federal de São Paulo (Escola Paulista de M edicina e Hospital São Paulo) em 4 categorias: funcionários, alunos, docentes e enfermeiros. A distribuição dos questionários foi feita às secretárias dos Departamentos e/ou Disciplinas e/ou Setores, para que fossem entregues aos membros de cada local e após 2 ou 3 dias, em média, os questionários eram recolhidos por um de nossos monitores. Os alunos da UNIFESP preencheram os questionários nos intervalos das aulas.

Foi aplicado um questionário confidencial, auto-explicável e auto-respondível, contendo 51 questões de múltipla escolha, dividido em três partes: a primeira avaliava o comportamento em relação ao fumar no trabal ho; a segunda, respondida apenas pelos fumantes, avaliava o envolvimento com o cigarro, tentativas em parar de fumar, a identificação do estágio de motivação para parar de fumar; a terceira, respondida apenas pelos ex-fumantes, abordava como era a dependência pelo cigarro. Os resultados foram analisados pelo SPSS 4 e a significância da diferença entre duas categorias foi avaliada pelo teste do Qui-quadrado, sendo considerado o limite de 5\% para rejeição da hipótese de nulidade ${ }^{13}$.

\section{RESULTADOS}

Obteve-se um total de $2.613(48,6 \%)$ de questionários respondidos. Do grupo de funcionários conseguiu-se obter 1.236 questionários respondidos, $37,3 \%$ do total de funcionários, sendo $26,5 \%$ do sexo mascul ino e $73,5 \%$ do sexo femi ni no. Do grupo de alunos, obtivemos 914 questionários respondidos, $76,5 \%$ do total de alunos, sendo 514 (78\% do total) do Curso de M edicina, 261 (100\% do total) de Enfermagem, 68 (48\% do total) de Biomédica e 98 (93\% do total) de Fonoaudiologia, com 37\% de al unos do sexo masculino e $63 \%$ do sexo feminino. Do grupo de docentes, 326 (49\%) questionários foram respondidos, sendo $54,6 \%$ do sexo masculino e $45,4 \%$ do sexo femi nino. Do grupo de enfermeiros (as) obtivemos $52,7 \%$ de questionários respondidos, sendo $4,8 \%$ do sexo masculino e $94,2 \%$ do sexo feminino (Tabela 1 ).

A média de idade da população total foi de 32,6 anos. A média de idade encontrada para os fumantes foi de 34,9 anos, para os ex-fumantes de 36,9 anos e para os não-fumantes de 31,2 anos. A prevalência total de fumantes foi de $15,5 \%$ sendo $15 \%$ para o sexo masculino e $16 \%$ para o sexo feminino. As prevalências de fumo por categorias encontram-se no Figura 1. A idade onde prevalece maior número de indivíduos fumantes foi entre 31 e 40 anos de idade $(26,6 \%)$ e a prevalência de exfumantes cresce com a idade, chegando a $45,5 \%$ de 
Tabela 1 - Percentual de questionários respondidos e média da população estudada na UNIFESP, 1996

\begin{tabular}{lccccc} 
& total de pessoas & \multicolumn{2}{c|}{ \% de questionários respondidos } & sexo feminino \\
\cline { 3 - 6 } & & total & sexo masculino & sexia de idade \\
Funcionários & 3.313 & $1.236(37,3)$ & $327(26,5)$ & $909(73,5)$ & 36,4 \\
Alunos & 1.144 & $914(76,5)$ & $338(37,0)$ & $576(63,0)$ & 22,0 \\
Docentes & 665 & $326(49,0)$ & $178(54,6)$ & $148(45,4)$ & 45,0 \\
Enfermeiros & 260 & $137(52,7)$ & $7(4,8)$ & $130(95,2)$ & 32,6 \\
Total & 5.810 & $2.613(48,6)$ & $892(33,0)$ & $1.803(67,0)$ & 32,6 \\
\hline
\end{tabular}

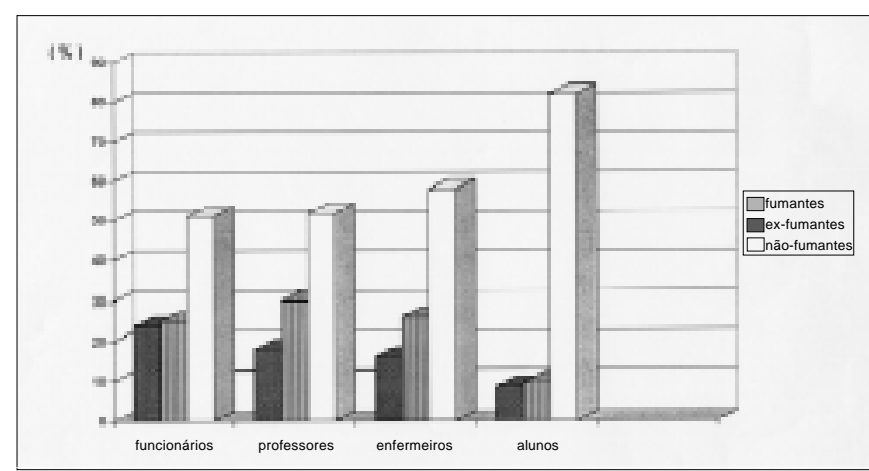

Fig. 1 - Fumantes, ex-fumantes e não-fumantes, por categorias, UNIFESP, 1996.

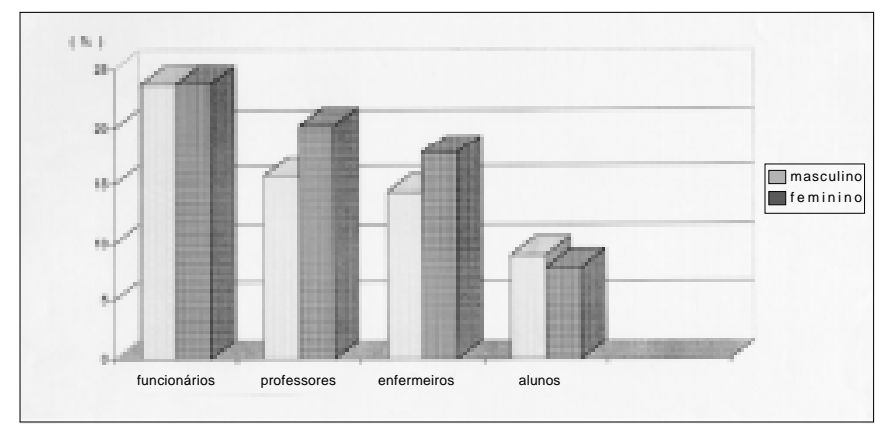

Fig. 2 - Prevalência de fumantes, por sexo e categorias, UNIFESP, 1996.

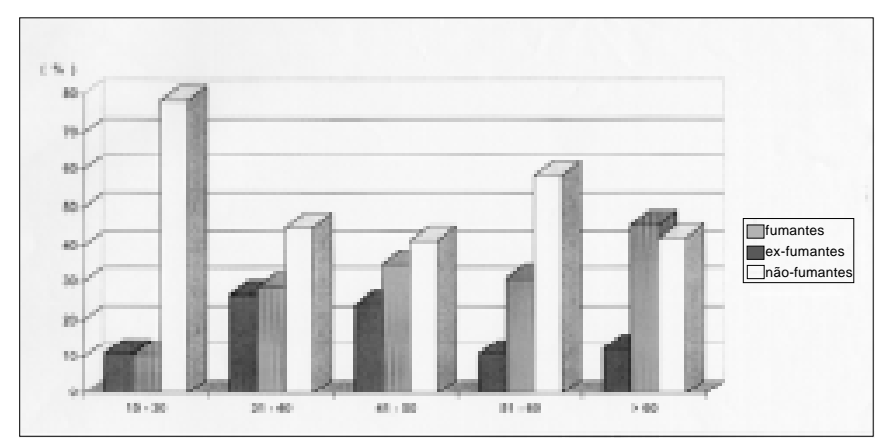

Fig. 3 - Prevalência de fumantes, ex-fumantes e nãofumantes, por faixas etárias, UNIFESP, 1996

ex-fumantes nos indivíduos com mais de 60 anos (Fig. 3). Cinquenta e nove porcento dos indivíduos que responderam o questionário disseram não ter pessoas fumantes em casa, enquanto que $40,6 \%$

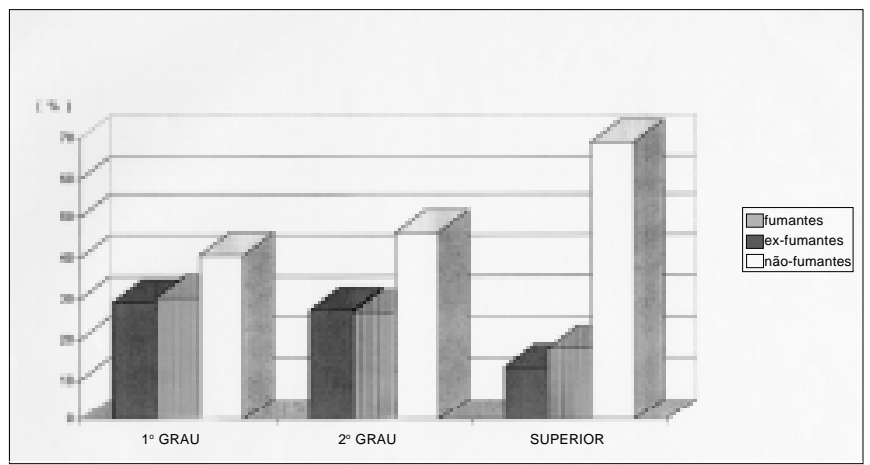

Fig. 4 - Prevalência de fumantes, ex-fumantes e nãofumantes, por nível de instrução, UNIFESP, 1996

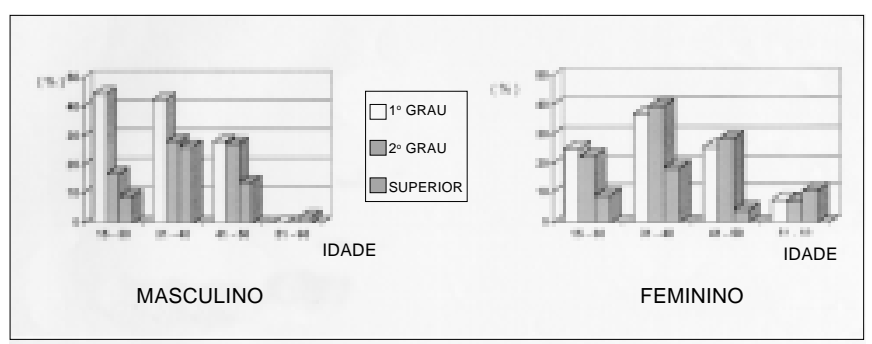

Fig. 5 - Prevalência de fumantes, por idade e nível de instrução, em ambos os sexos, UNIFESP, 1996.

tinham indivíduos fumantes em casa. Dos que responderam afirmativamente a este quesito, $65 \%$ deles só tinham um fumante em casa, $25 \%$ dois fumantes e $10 \%$ mais de dois fumantes. A prevalência de fumantes por idade e sexo com relação ao grau de instrução pode ser observada na Figura 5, a porcentagem de fumantes por sexo e categoria na Figura 2 e a porcentagem de fumantes por nível de instrução na Figura 4.

A preocupação com o fumo passivo foi expressada por $82,5 \%$ das pessoas e $71,4 \%$ da população total se incomoda pelo cigarro fumado pelos outros, sendo o cheiro da fumaça o mais citado, segui do pela irritação nos ol hos e nariz e sensação de falta de ar após a inalação passiva da fumaça. Quarenta e cinco porcento dos fumantes descreveram sintomas relacionados ao fumo.

Do total de indivíduos fumantes da UNIFESP $59 \%$ já tentaram parar e não conseguiram, 55\% 
Tabela 2 - Idade de início, quantidade e intensidade do tabagismo na UNIFESP, 1996.

\begin{tabular}{|lccc|}
\hline & Idade de início & $\mathbf{N}^{\circ}$ de cig./dia & Anos/maço \\
Fumantes & 19,1 & 12,2 & 14,0 \\
Ex-fumantes & 20,8 & 19,1 & 17,9 \\
\hline
\end{tabular}

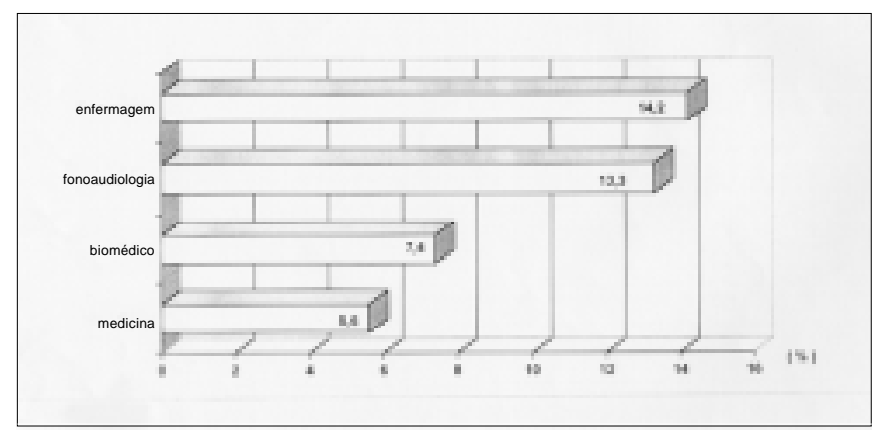

Fig. 6 - Porcentagem de fumantes entre alunos de diferentes cursos, UNIFESP, 1996.

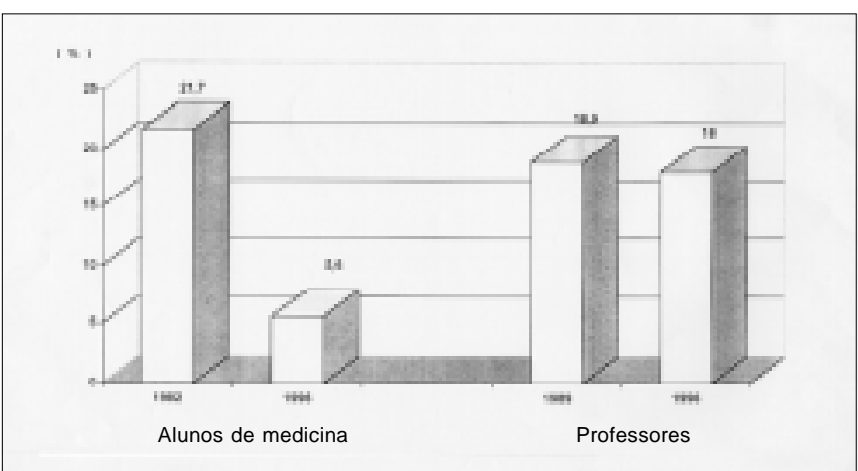

Fig. 7 - Comparação entre as prevalências de fumantes em estudantes de medicina e professores universitários em datas diferentes, UNIFESP.

gostariam de parar de fumar e $42 \%$ gostariam de al guma ajuda para parar de fumar. As características da idade de início do consumo de cigarros, quantidade e tempo de fumo estão mostradas na Tabela 2.

Na população de funcionários os fumantes representaram 23,7\% da população total , não havendo diferença entre o percentual de fumantes por sexo e a média de idade foi de 36,4 anos. Os exfumantes representaram $25,1 \%$ da população total ( $27,5 \%$ entre homens e $24,4 \%$ entre as mulheres) e os não-fumantes foram $51,2 \%$ da população. $\mathrm{Na}$ opinião dos funcionários em relação a fumar no ambiente de trabalho $62,4 \%$ acham que deveriam haver áreas restritas e $29 \%$ acham que deveria ser totalmente proibido. A maior prevalência de fumantes entre funcionários foi encontrada nos indivíduos com primeiro grau (33\%) e a menor nos funcionários com nível superior (18,7\%). Dos fumantes, $85,7 \%$ admitiram fumar no ambiente de trabal ho e $89,8 \%$ no ambiente domiciliar. Foi respondido que $40,9 \%$ dos funcionários fumantes pretendiam parar de fumar em 6 meses e 54\% gostariam de obter algum tipo de ajuda para parar de fumar.

Dos 1.195 alunos matriculados no ano de 1995 nos cursos da UNIFESP, a média de idade foi de 22 anos. A porcentagem de fumantes, por curso, encontra-se na Figura 6 . Dentre $8,6 \%$ de alunos fumantes, $27 \%$ responderam que pretendem parar de fumar em 6 meses. Não houve diferença estatisticamente significante entre as prevalências de fumantes nos diferentes anos de um mesmo curso. A média de idade dos docentes foi de 45 anos. Destes, $18 \%$ são fumantes, $30 \%$ são ex-fumantes e $52 \%$ são não-fumantes. Dos fumantes, $57,9 \%$ são do sexo masculino e $42,1 \%$ do sexo feminino. Quanto ao que pensam sobre fumar no ambiente de trabalho, 51\% acham que deveria ser permitido em áreas restritas e 35\% são a favor da proibição total do fumo no ambiente de trabalho.

Noventa e cinco porcento dos docentes fumantes já tentaram parar de fumar pelo menos uma vez, e $39,3 \%$ estão pensando seriamente em parar de fumar nos próximos 6 meses. Entre as enfermeiras encontrou-se um percentual de $16 \%$ de fumantes. Das fumantes, 67,5\% manifestaram-se a favor de fumar em áreas restritas e $32 \%$ a favor da proibição total do fumo no ambiente de trabal ho. Dentre as enfermeiras, 30\% manifestaram-se predispostas a parar de fumar nos próximos 6 meses.

\section{DISCUSSÃO}

No Brasil assiste-se a uma invasão tabágica, cuja epidemia se aguçou a partir de 1970. Desse ano até 1990 , enquanto a população cresceu $61,5 \%$ o consumo de cigarros aumentou 125,7\%. Levantamento feito pelo IBGE em 1989 estimou em quase 31 mi lhões o número de tabagistas, representando $32,6 \%$ da população acima dos 15 anos. Do total de fumantes $40,4 \%$ são mulheres. Dos 15 aos 19 anos temos 2,4 milhões de fumantes ( $75 \%$ dos fumantes começam entre 10 e 18 anos) $)^{7,9}$. Carecemos de estatísticas mais confiáveis, mas registrando a mortalidade por doenças para as quais o tabaco é significante fator de risco e o número per capita do consumo de cigarros, pode-se estimar que morrem aqui 80.000 a 120.000 tabagi stas por ano, constituindo $13 \%$ a $16 \%$ da mortalidade geral, o que é um al to índice?

Com relação ao sexo, observa-se mundialmente a tendência de diminuição da prevalência de fumantes 
em ambos os sexos, porém esta diminuição é mais lenta no sexo feminino, o que faz com que nesta década, em muitos dos trabalhos de prevalência de tabagismo, os números encontrados sejam iguais entre homens e mulheres ${ }^{2,9}$. Nas grandes ci dades brasil eiras o número de mul heres fumantes tem sido igual ou até maior que o dos homens, como se verifica no nosso trabalho dentre os fumantes na faixa etária de 41 a 50 anos. Verificamos também que a prevalência de homens exfumantes encontrada foi maior no sexo masculino que no sexo feminino. A faixa etária em que mais se fuma para ambos os sexos no Brasil é a de 30 a 49 anos, fato este verificado na população estudada (Figura 3). Com relação ao nível de instrução os indivíduos com menor grau de instrução têm maior prevalência de tabagismo (Figura 4).

Dentre os al unos de medicina da UNIFESP, a de prevalência de tabagismo era de $21,7 \%$ em $1982^{14}$, sendo agora de 5,6\%. Em alunos de medicina de Ribeirão Preto, Ruffino-Netto verificou uma prevalência de $27,7 \%$ em 1980 e $14,5 \%$ em $1986^{15}$ e Rosemberg, em acadêmicos de medicina de Sorocaba, verificou $37,8 \%$ de prevalência no ano de 1979, 17,7\% em 1990, e 1,3\% em 1996, refletindo, portanto, uma significativa taxa de redução de tabagismo nesta população ${ }^{16}$.

A pesar das indústrias do tabaco visarem adquirir jovens adeptos do cigarro através de anúncios apelativos, propagandas de televisão, patrocínio de shows e grandes eventos esportivos dirigidos ao público jovem, parece que os jovens universitários da área de saúde têm ti do bom nível de informação sobre os mal efíci os do fumo através de proibições e campanhas educativas, conseguindo, desta forma, não se transformarem em fumantes.

Comparando-se os dados obtidos em pesquisa realizada com 399 docentes da U nifesp no ano de 1988, verificou-se que $18,9 \%$ dos docentes eram fumantes (19,4\% dos sexo masculino e $18,4 \%$ do sexo feminino); $25,8 \%$ eram ex-fumantes (36,4\% do sexo masculino e $15,1 \%$ do sexo feminino) e $55,4 \%$ não-fumantes (44,2\% do sexo masculino e $66,5 \%$ do sexo femi nino). Oitenta por cento começou a fumar antes dos 20 anos de idade e dentre os ex-fumantes $84,1 \%$ deixaram de fumar abruptamente, sendo que em $86,2 \%$ dos casos não foi utilizado nenhuma metodologia especial para abandono do fumo. Os efeitos secundários relatados pelos ex-fumantes após terem deixado de fumar foram: ganho de peso $(53,5 \%)$, ansiedade $(15,4 \%)$, agitação $(8,1 \%)$, insônia $(7,1 \%)^{17}$.

Comparando-se o percentual de $18,9 \%$ de docentes fumantes em 1989 contra os 18\% de docentes fumantes em 1996, notamos uma redução de tabagismo relativamente pequena nesta população, considerando-se grau de instrução, a probabilidade destas pessoas já apresentarem al guns dos sintomas relacionados ao fumo, sobretudo respiratórios e a incorporação da bagagem cultural, adquirida pela experiência diária no convívio com pacientes, muitos com moléstias associadas ao tabagismo. I sto nos faz acreditar que a experiência pessoal na área de saúde, as campanhas publicitárias e educativas existentes no nosso país não afetaram os hábitos tabágicos da população de docentes universitários na UNIFESP, que continuam fumando cerca de 2 vezes mais quando analisamos os dados em confronto com as taxas de prevalência de fumantes em médicos americanos, por exemplo (Figura 7).

O Ministério da Saúde tem preconizado que se façam levantamentos periódicos, assim como se inicie programas educativos em todos os níveis que venham a corroborar com algumas das leis existentes no país, algumas antigas e outras mais recentes, como a de não se fumar em instituições públicas. A primeira de Lei de âmbito nacional (no. 9294/96) que “di spõe sobre as restri ções ao uso e à propaganda de produtos fumígeros, bebidas alcoólicas, medicamentos, terapias e defensivos agrícolas, nos termos do parágrafo 4․ da Constituição F ederal", teve grande repercussão na mídia e na população e tem grande efetividade educativa quanto à nocividade do tabaco. No entanto, várias brechas na Lei precisarão ser revistas, como não prever multas para os infratores tabagistas e responsáveis pelos estabelecimentos onde se fuma, permitir fumar em recintos fechados em locais especialmente destinados para este fim e proibir fumar em salas de aula, deixando livre desta proibição outros recintos escol ares.

Acreditamos, por fim, que as campanhas educativas sobre tabagismo poderiam ser realizadas e reforçadas em nível local, em instituições públicas e privadas, abolindo a exposição ao tabaco, impulsionando assim uma grande campanha de apoio mútuo entre ex-fumantes, não-fumantes e fumantes, para tornar o ambiente de trabalho livre da fumaça. Certamente estas medidas se expandiriam também para o ambiente domiciliar, fazendo com que principalmente as crianças crescessem livre da fumaça do tabaco, assistindo a bons exemplos e não experimentando os malefícios do fumo desde tenras idades.

$\mathrm{N}$ ão acreditamos que medidas proi bitivas, tidas como parciais, possam dar resultados na diminuição da prevalência dos fumantes quando os i solam em recintos específicos, geralmente com pouca ventilação, para fumar. Neste caso, os malefícios 
causados aos não-fumantes serão praticamente os mesmos de ficarem no mesmo ambi ente do fumante, pois a fumaça do tabaco se espal ha rapi damente por todo o ambiente. As medi das mais abrangentes de cunho educativo, com restrições do fumo em alguns locais apropriados e com proibição total do fumo posteriormente, tendem a ser as mais aceitas, associadas a medidas de suporte para quem pretende parar de fumar ${ }^{18,19}$.

\section{SUMMARY}

\section{Tobacco smoking at Federal University of São Paulo (UNIFE SP), Brazil, 1996. Prelimi- nary data of an institutional program.}

Purpose. To evaluate the prevalence of tobacco smoking among health workers, professores, nurses and students of Federal University of São Paulo and the acceptance of an institutional program for quitting smoking.

MetHODS. We analized the answers of a questionnairy with 51 questions, distributed to people from different categories.

RESULTS. The total percentage of answered questionnaires was $48.6 \%$ (2613). The answers obtained from heal th workers were $37.3 \%$, professors $49.0 \%$, nurses $52.7 \%$ and students $76.5 \%$ The total percentage of smokers at UNIFESP was $15.5 \%$ : $23.7 \%$ for health workers, $18 \%$ for professors, $16 \%$ for nurses and $8.6 \%$ for students. There was no significant statistical difference between the prevalence of smoking among females (17.3\%) and males (16.3\%). The age of major prevalence of smoking was between 31 to 40 years (26.6\%). For all ages, people who have university level smoked less, independent of sex. Eighty three percent $(83 \%)$ of the UNIFESP workers and students are worried of being passive smokers. Between the smokers, $55 \%$ were thinking of quiting and $42 \%$ declared that need some hel $p$ to quit.

Conclusion. We concluded that educational programs and help for cessation at institutional level are necessary and well accepted at UNIFESP, and the completion of these programs will contribute to the obeying of the prohibitive laws of no smoking within the community. [Rev Ass Med Brasil 1999; 45(1): 39-44.]

KEY WORDS: Tobacco smoking. Prevalence. Health workers. Professors, students and nurses.

\section{REFERÊNCIAS BIBLIOGRÁFICAS}

1. US Public Health Service: Smoking and Health. A Report of the Advisory Committee to the Surgeon General of the Public
Health Service. PHS Publication No 1103. Washington, DC, US Government Printing Office, 1964.

2. US Department of Health and Human Services: Smoking, Tobacco and Cancer Program 1985-1989 Status Report. US Department of Health and Human Services, Public Health Service, National I nstitutes of H ealth, National Cancer I nstitute, NIH Publication No90-3107, 1990.

3. US Department of $\mathrm{Health}$ and Human Services: Reducing the health consequences of smoking: 25 years of progress. A Report of the Surgeon General. US Department of Health and Human Services, Public Health Service, Centers for Disease Control, Center for Chronic Disease Prevention and Health Promotion, Office on Smoking and Health. DHHS Pub. No. (CDC) 89-8411, 1989.

4. Harvey, L., Stellman, S.D.. Public opinion on health issues. In: Surveys of physician and Public Opinion on Health Care I ssues. Chicago, American Medical Association, 1987.

5. Sachs, D. P. L.. Smoking habits among pulmonary physicians. N. Engl. J . Med. 1983,309:799.

6. Remington, P.L., Forman M.R., Gentry, E.M., Marks, J .S., Hogelin, G.C., Trowbrigde, F.L.. Current smoking trends in the United States. J AMA 1985;253:2975-8.

7. IBGE. Pesquisa Nacional sobre Saúde e Nutrição: dados preliminares. Rio de J aneiro, 1989.

8. Rosemberg, J .. A propósito do Inquérito Piloto da AMB sobre otabagismoentremédicos. Rev. Ass. Med. Brasil. 1983,29:127.

9. Ministério da Saúde. Instituto Nacional de Câncer. Controle do tabagismo: um desafio. Rio de J aneiro, p. 12-13, 1991.

10. Boletim da Secretaria de Estado de Saúde (CVE - Centro de Vigilância E pidemiológica - Divisão de Doenças crônicas nãotransmissíveis) - Gastos hospitalares com doenças tabacoassociadas, 1995.

11. Chaieb, J. A., Fasolo, P., Ruschel, S. P., Costa, J. T. F.. Aspectos epidemiológicos e tabagismo em Porto Alegre: Prevalência do tabagismo e DPOC. J. Pneumol. 1995; 21(4):171-9.

12. Organização Mundial da Saúde. Strategie de lutte antitabac dans les pays en devel oppement. Série de I nformes Técnicos. Genebra, 1983.

13. Siegel, S. Estadística no parametrica aplicada a las ciências dela conducta. $2^{\text {a }}$ ed. México, Trillas, 1975.

14. Nunes, V. A. Contribuição ao estudo do tabagismo, dos sintomas edoenças pul monares em popul ações uni versitárias vivendo em ambientecom níveis diferentes depol uição atmosférica. Tese apresentada à Escola Paulista de Medicina. Doutorado. 1986. 103p.

15. Ruffino-N etto, A., Ruffino, M. C., Gruber, C. A., I zuzuki, E. E., Carramaschi, F. R.. Tabagismo entre acadêmi cos de Ribeirão Preto - SP. J ornal dePneumologia, 1981, 7:164.

16. Rosemberg, J ., Peron, S.. Tabagismo entre estudantes da F aculdade de Ciências Médicas de Sorocaba. Tabagismo nos acadêmicos de medicina e nos médicos. J ornal de Pneumologia 1990 16(1):13-22.

17. Maria I sabel Sampaio Carmagnani - Monografia - Eliminação do hábi to defumar. Subsídios para elaboração de programas educativos. São Paulo. UNIFESP, 1989.

18. Laranjeira, R., Ferreira M. P.. Comocriar um hospital livrede cigarros. Rev. Ass. Med. Brasil. 1997,43(2):169-72.

19. Petty, T.L., N ett, L.M.. How to hel p your patient stop smoking - What works and what doesn't work. Seminars in respiratory and critical care medicine 1995, 16(2). 